\title{
THE NONLINEAR OSCILLATIONS OF A STRING*
}

By A. G. MACKIE (Institute for Fluid Dynamics and Applied Mathematics, University of Maryland)

This note is intended to shed further light on an observation by Rosen [1] concerning papers by Zabusky [2] and Kruskal and Zabusky [3] in which he shows that the nonlinear vibrations of a string considered in these papers are formally equivalent to solutions of the one-dimensional equations of motion of a polytropic gas in homentropic flow. We show here that the equivalence is more than formal and that the equation (4) below governing the motion of a string considered in [2] and [3] is exactly the same as that governing the motion of a gas with a certain equation of state, provided Lagrangian co-ordinates are used to describe the motion of the gas. By having the equivalence linked in this way through the Lagrangian representation, Rosen's observation is motivated physically and may in fact be generalized.

We consider a gas in one-dimensional motion with $p$, the pressure, a given function of $\rho$, the density. Let $x$ be the length co-ordinate measured in the given direction and let $y(x, t)$ be the longitudinal displacement at time $t$ of a particle of gas which at $t=0$ was at position $x$. Let us also suppose for convenience that $p=p_{0}, \rho=\rho_{0}$ for all $x$ when $t=0$, the suffix zero denoting a constant state. Then it is easy to show that the continuity equation gives

$$
\rho(1+\partial y / \partial x)=\rho_{0}
$$

and that the equation of motion is

$$
\rho_{0}\left(\partial^{2} y / \partial t^{2}\right)=-\partial p / \partial x
$$

If $c^{2}=d p / d \rho$, then the equation of motion is

$$
\partial^{2} y / \partial t^{2}=\left(\rho^{2} c^{2} / \rho_{0}^{2}\right)\left(\partial^{2} y / \partial x^{2}\right)
$$

from (1), or alternatively

$$
\partial^{2} y / \partial t^{2}=f(1+\partial y / \partial x)\left(\partial^{2} y / \partial x^{2}\right),
$$

since $c^{2}$ is a function of $\rho$. For a polytropic gas in which $p \propto p^{\gamma}, \rho^{2} c^{2} \propto \rho^{\gamma+1}$ and

$$
\partial^{2} y / \partial t^{2}=K(1+\partial y / \partial x)^{-\gamma-1}\left(\partial^{2} y / \partial x^{2}\right),
$$

where $K$ is some positive constant. Trivial scaling changes of variables now enable the equation to be reduced to that studied in [2] and [3], namely

$$
\partial^{2} y / \partial t^{2}=(1+\epsilon(\partial y / \partial x))^{\alpha}\left(\partial^{2} y / \partial x^{2}\right) .
$$

This approach also leads to a generalization of Rosen's analogy. For a gas which is nonpolytropic but which has an acceptable equation of state, the acoustic impedance $\rho c$ is a decreasing function of $1 / \rho$. Accordingly, from (1) and (2), we may consider strings with an equation of motion of the general form (3) provided $f^{\prime}<0$ and still be able to analyze their vibrations by means of the conventional apparatus of one-dimensional gas dynamics.

*Received October 27, 1966. This work was supported in part by the Air Force Office of Scientific Research under Grant or Contract AF 1194-67. 
We may summarize as follows. Any problem for a vibrating string, whose longitudinal displacement is governed by Eq. (3), is equivalent to a problem in the dynamics of a gas with a given pressure-density relationship obtained by integrating

$$
d p / d \rho=\left(\rho_{0} / \rho\right)^{2} f\left(\rho_{0} / \rho\right)
$$

with $p=p_{0}$ when $\rho=\rho_{0}$. When the gas is polytropic the equation reduces to (4). The equivalence is precise in the sense that the longitudinal displacement of the string is identified with the longitudinal displacement (or Lagrangian co-ordinate) of the gas. Zabusky does remark at the end of Sec. 2 of [2] that Eq. (3) is that which governs the Lagrangian of a nonlinear, hysteresis-free, solid continuum but does not pursue the point in order to use directly the well-established Eulerian procedures in gas dynamics to supply information on the behavior of the nonlinear string. Rosen, on the other hand, shows by an inverse process that Zabusky's equation is formally equivalent to the Eulerian equations of gas dynamics but does not explicitly identify the Lagrangian coordinate with the longitudinal displacement of the string. It is hoped that this note will help to clarify and unify the different lines of thought.

\section{REFERENCES}

[1] G. Rosen, Formal equivalence of the non-linear string and one-dimensional fluid flow, Quart. Appl. Math. 23, 286-287 (1965)

[2] N. J. Zabusky, Exact solution for the vibrations of a non-linear continuous model string, J. Math. Phys. 3, 1028-1039 (1962)

[3] M. D. Kruskal and N. J. Zabusky, Stroboscopic-perturbation procedure for treating a class of non-linear wave equations, J. Math. Phys. 5, 231-244 (1964) 